\title{
In situ green synthesis of antimicrobial carboxymethyl chitosan-nanosilver hybrids with controlled silver release
}

This article was published in the following Dove Press journal:

International Journal of Nanomedicine

I8 April 2017

Number of times this article has been viewed

\section{Siqi Huang \\ Zhiming Yu \\ Yang Zhang \\ Chusheng Qi \\ Shifeng Zhang}

MOE Key Laboratory of Wooden Material Science and Application, Beijing Forestry University, Beijing, China
Correspondence: Zhiming Yu; Yang Zhang MOE Key Laboratory of Wooden Material Science and Application, Beijing Forestry University, 35 Qinghua East Road, Haidian, Beijing 100083, China $\mathrm{Tel} / \mathrm{fax}+861062337751$;

+861062336907

Email yuzhiming@bjfu.edu.cn; bjfuzhangyang@bjfu.edu.cn
Abstract: In order to fabricate antimicrobial carboxymethyl chitosan-nanosilver (CMC-Ag) hybrids with controlled silver release, this study demonstrated comparable formation via three synthetic protocols: 1) carboxymethyl chitosan (CMC) and glucose (adding glucose after $\mathrm{AgNO}_{3}$ ), 2) $\mathrm{CMC}$ and glucose (adding glucose before $\mathrm{AgNO}_{3}$ ), and 3) $\mathrm{CMC}$ only. Under principles of green chemistry, the synthesis was conducted in an aqueous medium exposed to microwave irradiation for 10 minutes with nontoxic chemicals. The structure and formation mechanisms of the three CMC-Ag hybrids were explored using X-ray diffraction, ultravioletvisible spectroscopy, transmission electron microscopy, and Fourier-transform infrared analyses. Additionally, antimicrobial activity and in vitro silver release of the three synthesized hybrids were investigated in detail. The results revealed that a large number of stable, uniform, and small silver nanoparticles (AgNPs) were synthesized in situ on CMC chains via protocol 1. AgNPs were well dispersed with narrow size distribution in the range of 6-20 $\mathrm{nm}$, with mean diameter only $12.22 \pm 2.57 \mathrm{~nm}$. The addition of glucose resulted in greater AgNP synthesis. The order of addition of glucose and $\mathrm{AgNO}_{3}$ significantly affected particle size and size distribution of AgNPs. Compared to CMC alone and commercially available AgNPs, the antimicrobial activities of three hybrids were significantly improved. Of the three hybrids, CMC-Ag1 synthesized via protocol 1 exhibited better antimicrobial activity than CMC-Ag2 and CMC-Ag3, and showed more effective inhibition of Staphylococcus aureus than Escherichia coli. Due to strong coordination and electrostatic interactions between $\mathrm{CMC}$ and silver and good steric protection provided by $\mathrm{CMC}, \mathrm{CMC}-\mathrm{Ag} 1$ displayed stable and continuous silver release and better performance in retaining silver for prolonged periods than CMC-Ag2 and CMC-Ag3.

Keywords: carboxymethyl chitosan/nanosilver hybrids, green synthesis, formation mechanism, controlled silver release, antimicrobial effect

\section{Introduction}

Health issues caused by microbial contamination have troubled human beings for many years. ${ }^{1-3}$ Over the past few decades, the excessive use of antibiotics has allowed bacteria to develop resistance from continual exposure to conventional antibiotics. ${ }^{4}$ The problem of decreasing effectiveness of our existing antibiotics is now recognized as a significant worldwide issue, and a new generation of antimicrobial agents to replace antibiotics is being researched and explored. ${ }^{5-7}$

Silver nanoparticles (AgNPs) present an advantageous alternative to antibiotics, as they have efficient antimicrobial activity toward a broad range of microorganisms, but are unlikely to induce bacteria to develop resistance, due to their multifaceted and complex antimicrobial mechanisms. ${ }^{8-12}$ Products containing nanosilver are 
being incorporated into consumer goods, including textiles, coatings, food packaging, antiseptic sprays, bandages, and biomedical implants. ${ }^{13,14}$ With increased production volume have come concerns about potential adverse effects on the environment and human health, because many products release large amounts of superfluous silver as soluble ions during initial use or washing. ${ }^{14-17}$ This rapid release of silver may also result in only short-term antimicrobial performance. ${ }^{18}$ Therefore, there is great need for and significant interest in optimizing the formulations of nanosilver to achieve controlled silver release.

Polysaccharide-nanosilver hybrids have attracted recent attention. ${ }^{19-22}$ The presence of polysaccharide allows chelation or adsorption, which acts as a stabilizer to prevent aggregation of NPs and facilitates the continuous release of silver. ${ }^{23,24}$ Carboxymethyl chitosan (CMC) is a novel amphoteric biopolymer synthesized by grafting carboxymethyl groups onto chitosan (an amino polysaccharide derived from natural chitin) chains. Favored for its properties of biocompatibility, biodegradability, antimicrobial effect, and good water-solubility over a large range of $\mathrm{pH}$ conditions, $\mathrm{CMC}$ is widely applied in drug encapsulation, tissue engineering, antioxidant, and cosmetic and antibacterial fields. CMC possesses a large number of ammonium groups, carboxyl groups, and hydroxyl groups, and has a network-like structure that is considered a promising green stabilization agent for the preparation of nanometals. Long et al synthesized AgNPs by using commercially available CMC and sunlight, but this process was time-consuming and the resulting AgNPs were around $100 \mathrm{~nm}$ in size. ${ }^{25}$ In an effort to prepare small AgNPs rapidly, An et al used CMC as a stabilizer, but an environmentally harmful reducing agent, $\mathrm{NaBH}_{4}$, was required. ${ }^{26}$ Glucose is the most abundant natural monosaccharide, and its free-aldehyde group makes it a remarkable nontoxic reducing agent for producing nanometals. ${ }^{19}$ In this study, an attempt was made to prepare green, facile, and efficient $\mathrm{CMC}-$ nanosilver (CMC-Ag) hybrids through in situ synthesis of nanosilver in water-soluble CMC matrices with environmentally benign glucose via microwave-irradiation method.

The rapid development of various laboratory protocols for the synthesis of nanocomposite materials requires a deeper understanding of the reaction mechanisms underlying the synthesis procedures. ${ }^{22}$ Prior to this study, relatively few reports have appeared on the synthetic mechanism required to form a CMC-Ag hybrid using glucose as the reducing agent. To address this need, this study demonstrates comparable formation of CMC-Ag hybrids via three synthetic protocols: 1) synthesis of $\mathrm{CMC}-\mathrm{Ag}$ hybrids with $\mathrm{CMC}$ and glucose (adding glucose after $\mathrm{AgNO}_{3}$ ), 2) synthesis of $\mathrm{CMC}-\mathrm{Ag}$ hybrids with $\mathrm{CMC}$ and glucose (adding glucose before $\mathrm{AgNO}_{3}$ ), and 3) synthesis of CMC-Ag hybrids with CMC only. X-ray diffraction (XRD), ultraviolet (UV)-visible spectroscopy, transmission electron microscopy (TEM), and Fourier-transform infrared (FT-IR) analyses were used to determine the reaction mechanisms of the three CMC-Ag hybrids, and the effects of glucose on the formation of AgNPs, including the amount, size, and size distribution were explored. Moreover, the antimicrobial activity and in vitro silver release of the three synthesized hybrids were investigated to identify the optimum protocol for producing the best antimicrobial hybrid with controlled silver release.

\section{Materials and methods}

All chemicals in this study were of analytical grade and used as received without further purification. $\mathrm{CMC}, \mathrm{AgNO}_{3}$, and glucose were used for the synthesis of CMC-Ag hybrids. CMC (molecular weight $100 \mathrm{kDa}$, degree of deacetylation $90.5 \%$, degree of substitution of carboxymethyl groups 0.81 ) was obtained from Zhejiang Biochemical (Taizhou, China). $\mathrm{AgNO}_{3}$ was sourced from Guanghua Science and Technology Co Ltd (Guangzhou, China). Commercially available AgNPs (particle size $<50 \mathrm{~nm}$, metal basis $99.5 \%$ ) used as a control in the antimicrobial test were purchased from Sigma-Aldrich (St Louis, MO, USA). All other reagents were obtained from Beijing Chemical Works (Beijing, China). In addition, an XH-100A microwave synthesis system was supplied by Xiang-Hu Technology Co Ltd (Beijing, China). Figure 1 shows the chemical structure of $\mathrm{CMC} .{ }^{27}$

\section{Preparation of carboxymethyl chitosan-nanosilver hybrids} Synthesis of CMC-Ag hybrid with CMC and glucose (adding glucose after $\mathrm{AgNO}_{3}$ )

CMC powder $(0.25 \mathrm{~g})$ was dissolved in $125 \mathrm{~mL}$ of distilled water to prepare a $2 \mathrm{mg} / \mathrm{mL}$ solution, then a few drops of

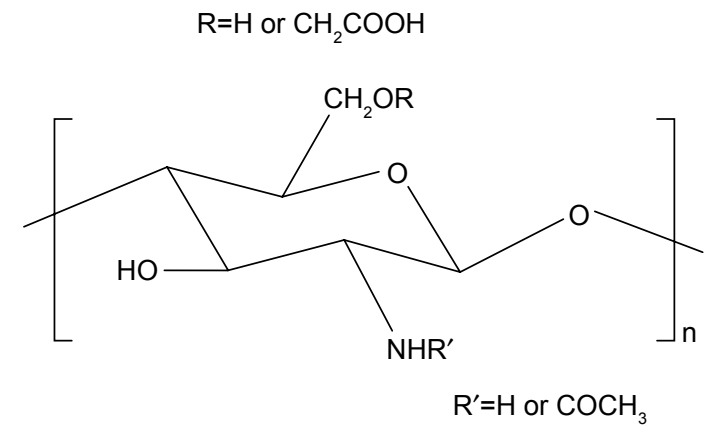

Figure I Chemical structure of carboxymethyl chitosan. 
diluted $\mathrm{NaOH}$ solution were added to adjust the $\mathrm{pH}$ to 9 . $\mathrm{AgNO}_{3}(0.17 \mathrm{~g})$ and glucose $(0.36 \mathrm{~g})$ were separately dissolved in distilled water to obtain $0.1 \mathrm{mmol} / \mathrm{mL}$ solutions. After that, the $\mathrm{AgNO}_{3}$ solution was added dropwise to the prepared $\mathrm{CMC}$ solution and reacted via microwave irradiation at $600 \mathrm{~W}$ at $80^{\circ} \mathrm{C}$ for 3 minutes. Subsequently, glucose solution was dropped slowly into the mixture and reacted for another 7 minutes using the same microwave-irradiation conditions. Next, the resulting reaction mixture was dialyzed against deionized water for 5 days. After precipitation and three washes with ethanol, the purified product was freezedried at $-60^{\circ} \mathrm{C}$ for 24 hours and then ground into powder. The resultant composite was designated CMC-Ag1.

\section{Synthesis of CMC-Ag hybrid with CMC and glucose (adding glucose before $\mathrm{AgNO}_{3}$ )}

CMC powder $(0.25 \mathrm{~g})$ was dissolved in $125 \mathrm{~mL}$ of distilled water to prepare a $2 \mathrm{mg} / \mathrm{mL}$ solution, then a few drops of diluted $\mathrm{NaOH}$ solution were added to adjust the $\mathrm{pH}$ to 9 . $\mathrm{AgNO}_{3}(0.17 \mathrm{~g})$ and glucose $(0.36 \mathrm{~g})$ were separately dissolved in distilled water to obtain $0.1 \mathrm{mmol} / \mathrm{mL}$ solutions, then the glucose solution was dropped slowly into the prepared $\mathrm{CMC}$ solution with stirring. After that, the $\mathrm{AgNO}_{3}$ solution was added dropwise to the mixture and reacted via microwave irradiation at $600 \mathrm{~W}$ at $80^{\circ} \mathrm{C}$ for 10 minutes. Next, the resulting reaction mixture was dialyzed against deionized water for 5 days. After precipitation and three washes with ethanol, the purified product was freeze-dried at $-60^{\circ} \mathrm{C}$ for 24 hours and then ground into powder. The resultant composite was designated CMC-Ag2.

\section{Synthesis of CMC-Ag hybrid with CMC only}

CMC powder $(0.25 \mathrm{~g})$ was dissolved in $125 \mathrm{~mL}$ of distilled water to prepare a $2 \mathrm{mg} / \mathrm{mL}$ solution, then a few drops of diluted $\mathrm{NaOH}$ solution were added to adjust the $\mathrm{pH}$ to 9 . $\mathrm{AgNO}_{3}(0.17 \mathrm{~g})$ was dissolved in distilled water to obtain a $0.1 \mathrm{mmol} / \mathrm{mL}$ solution, then this was added dropwise to the prepared CMC solution and reacted via microwave irradiation at $600 \mathrm{~W}$ at $80^{\circ} \mathrm{C}$ for 10 minutes. Next, the resulting reaction mixture was dialyzed against deionized water for 5 days. After precipitation and three washes with ethanol, the purified product was freeze-dried at $-60^{\circ} \mathrm{C}$ for 24 hours and then ground into powder. The resultant composite was designated CMC-Ag3.

\section{Characterization}

XRD patterns of the powder samples were obtained in the $2 \theta$ range from $5^{\circ}$ to $85^{\circ}$ on a D8 Advance (Bruker Optik
$\mathrm{GmbH}$, Ettlingen, Germany) equipped with $\mathrm{Cu}-\mathrm{K} \alpha$ radiation $(\lambda=0.15418 \mathrm{~nm})$ at ambient temperature. UV-visible spectra of the samples were obtained with a UV2310II spectrophotometer (Inesa, Shanghai, China). The analysis was performed in the range of 300-600 $\mathrm{nm}$ at room temperature.

The morphology and dispersion of the AgNPs were evaluated via TEM (HT7700; Hitachi, Tokyo, Japan). High-resolution TEM (JEM-2100F; Jeol, Tokyo, Japan) was used to investigate the microstructure of the AgNPs at an accelerating voltage of $200 \mathrm{kV}$. To prepare samples for TEM and high-resolution TEM characterization, freeze-dried CMC-Ag hybrid powders were dispersed in 50\% ethanol solution with sonication and dropped onto a copper-mesh grid and then dried in a vacuum-drying oven at $50^{\circ} \mathrm{C}$ for 10 minutes. Particle size and size distribution of the AgNPs were statistically analyzed using the software ImageJ, and at least 150 individual particles were analyzed for each CMC-Ag hybrid. FT-IR spectra of the samples were recorded using a Vertex 70v FT-IR spectrophotometer (Bruker) at a resolution of $4 \mathrm{~cm}^{-1}$ and scanning number of 32 from 400 to $4,000 \mathrm{~cm}^{-1}$ by the $\mathrm{KBr}$ pellet method.

\section{Antimicrobial activity testing}

Gram-positive Staphylococcus aureus and Gram-negative Escherichia coli (provided by the Chinese Academy of Sciences) were selected as model pathogenic bacteria to study the antimicrobial activity of the $\mathrm{CMC}-\mathrm{Ag}$ hybrids. Antimicrobial tests was performed based on our previous report. ${ }^{28,29}$ First, $S$. aureus and E. coli were separately adjusted by addition of sterile distilled water to obtain $10^{7} \mathrm{CFU} / \mathrm{mL}$ solutions. Next, antimicrobial solutions were diluted in phosphate buffer ( $\mathrm{pH}$ 7.2) to appropriate concentrations, and $10 \mathrm{~mL}$ of each sample was added to sterile petri dishes containing $10 \mathrm{~mL}$ of nutrient agar solution. The bacterial suspensions of $2 \mu \mathrm{L}$ were then inoculated on the prepared nutrient medium that contained the antimicrobial suspension, and the dishes were incubated at $37^{\circ} \mathrm{C}$. Minimum inhibitory concentration (MIC) values were determined after 24 hours of incubation. MIC is defined as the lowest concentration at which no bacterial colony is visible. ${ }^{28}$ All experiments were conducted in triplicate.

\section{In vitro silver-release study}

The in vitro silver-release study was performed using a previously described method, with modification. ${ }^{30,31}$ The amount of silver released from the CMC-Ag hybrids and the initial amount of silver in the hybrids were evaluated by inductively coupled plasma-optical emission spectrometry 
Table I Initial amount of silver in the hybrids and cumulative amount of silver released after 240 hours evaluated by ICP-OES analyses

\begin{tabular}{llll}
\hline $\begin{array}{l}\text { Sample } \\
\text { code }\end{array}$ & $\begin{array}{l}\text { Initial amount } \\
\text { of silver }(\mu \mathrm{g})\end{array}$ & $\begin{array}{l}\text { Cumulative release of silver } \\
\text { after 240 hours }\end{array}$ \\
\cline { 3 - 4 } & & $\begin{array}{l}\text { Released } \\
\text { amount }(\mu \mathrm{g})\end{array}$ & $\begin{array}{l}\text { Release } \\
\text { percentage }\end{array}$ \\
\hline CMC-AgI & 510 & 63 & 12.35 \\
CMC-Ag2 & 407 & 188 & 46.19 \\
CMC-Ag3 & 98 & 34 & 34.69 \\
\hline
\end{tabular}

Note: All experiments were conducted in triplicate.

Abbreviations: ICP-OES, inductively coupled plasma-optical emission spectrometry; CMC-Ag, carboxymethyl chitosan-nanosilver.

(ICP-OES; Optima 8000; PerkinElmer, Waltham, MA, USA). The cumulative release percentage of silver was obtained by dividing the cumulative amount of silver released at each sampling time point by the initial amount of silver in the samples. All experiments were conducted in triplicate.

To detect the cumulative amount of silver release, CMCAg1, CMC-Ag2, and CMC-Ag3 (10 mg) were separately immersed in $10 \mathrm{~mL}$ of phosphate buffer (pH 7.2) and incubated at $37^{\circ} \mathrm{C}$ under agitation $(300 \mathrm{rpm})$. At specific time points ( 1 , $3,6,12,24,48,96,144,192$, and 240 hours), samples were centrifuged, then $2 \mathrm{~mL}$ of the supernatants were removed for analysis and replaced by an equivalent volume of fresh media. The concentration (ppm) of silver in the release medium at the sampling time point was measured using ICP-OES analysis and then converted to the cumulative release amount $(\mu \mathrm{g})$. To determine the initial amount of silver in the hybrids, the samples of CMC-Ag1, CMC-Ag2, and CMC-Ag3 (10 mg) were separately weighed and mixed in solutions of $10 \mathrm{~mL}$ $\mathrm{HNO}_{3}(65 \%)$ and $1 \mathrm{~mL} \mathrm{H}_{2} \mathrm{O}_{2}(30 \%)$, followed by microwave treatment for 30 minutes. After digestion, the solutions were diluted to a constant volume $(25 \mathrm{~mL})$ for ICP-OES analysis. Experimental results are presented in Table 1.

\section{Results and discussion}

\section{Synthetic mechanism of carboxymethyl chitosan-nanosilver hybrids}

The proposed synthetic mechanisms of CMC-Ag1, CMC-Ag2, and CMC-Ag3 are shown in Scheme 1. It has been reported that the formation of metal crystals involves nucleation, growth, and aggregation processes..$^{32,33} \mathrm{In}$ the case of CMC$\mathrm{Ag} 1$, when $\mathrm{AgNO}_{3}$ solution was added to the $\mathrm{CMC}$ solution, the silver ions initially diffused into the CMC matrix, then they were uniformly and tightly anchored in the CMC-polymer network by many $-\mathrm{NH}_{2},-\mathrm{COOH},-\mathrm{OH}$, and $-\mathrm{NHCOCH}_{3}$ groups on the main chains of $\mathrm{CMC}$ via coordination and electrostatic interactions. ${ }^{34-36}$ When the glucose solution was added to the mixture, the absorbed silver ions were then reduced to silver atoms according to equation 1 :

$$
\mathrm{CH}_{2} \mathrm{OH}(\mathrm{CHOH})_{4} \mathrm{CHO}+\mathrm{Ag}^{+} \rightarrow \mathrm{CH}_{2} \mathrm{OH}(\mathrm{CHOH})_{4} \mathrm{COOH}+\mathrm{Ag}
$$

Those preformed silver atoms served as the seeds for the deposition of other reduced silver atoms, and many atoms coalesced together, leading to the in situ growth of AgNPs in the polymer network. ${ }^{37,38}$ The further growth and aggregation process can be hindered by steric hindrance, chelation, and electrostatic effects from the polymer network. ${ }^{39}$ In this way, a large number of small AgNPs were synthesized in situ on the CMC chains, and AgNPs were well dispersed throughout the $\mathrm{CMC}$ matrix with narrow size distribution.

According to the protocol for preparing CMC-Ag2, glucose solution was added before the $\mathrm{AgNO}_{3}$ solution. After $\mathrm{AgNO}_{3}$ solution had been dropped into the $\mathrm{CMC}$ and glucose mixture, silver ions were quickly surrounded by abundant glucose molecules, which might have inhibited the interaction between silver ions and CMC chains. Under microwave irradiation, silver ions were reduced to silver atoms, forming dispersed NPs in the presence of glucose. Due to a lack of chelation and electrostatic interactions, as well as steric protection, the aggregation process failed to cease in time, so numerous larger particles were produced and finally resulted in wide particle-size distribution.

The literature has documented the use of cellulose as a reducing agent in the synthesis of metal nanostructures by virtue of the presence of primary hydroxyl groups on the $\mathrm{C}_{6}$ position of the glucosidic rings. ${ }^{35}$ The oxidation of cellulose primary alcohol to aldehyde or carboxyl groups plays an important role in the reduction of metal ions. ${ }^{39,40}$ Without an additional reducing agent, $\mathrm{CMC}$ exhibits weak reducing capacity, relying on the partially uncarboxymethylated primary hydroxyl groups. Therefore, the silver content in CMC-Ag3 was significantly lower than that in CMC-Ag1 and $\mathrm{CMC}-\mathrm{Ag} 2$. Moreover, in the synthesis of CMC-Ag3, some of the functional groups in CMC were involved in silver-ion reduction, which might have weakened the adsorption and immobilization abilities of CMC itself, helping to promote the formation of slightly larger AgNPs compared to CMC-Ag1.

\section{Characterization of the synthesis of carboxymethyl chitosan-nanosilver hybrids}

XRD analysis is a simple and effective method to confirm the formation of metallic particles by examination of the 


\section{A}

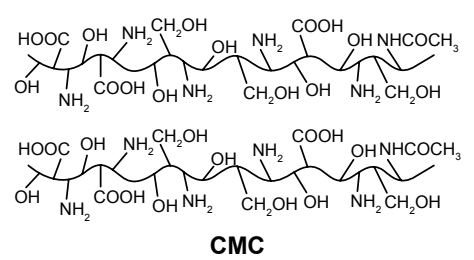

B

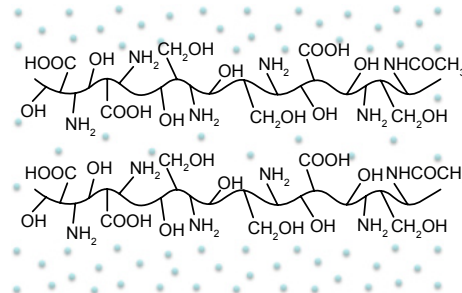

CMC \& glucose

\section{C}

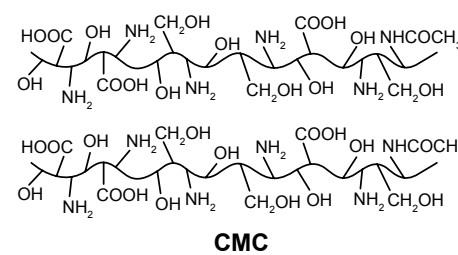

CMC
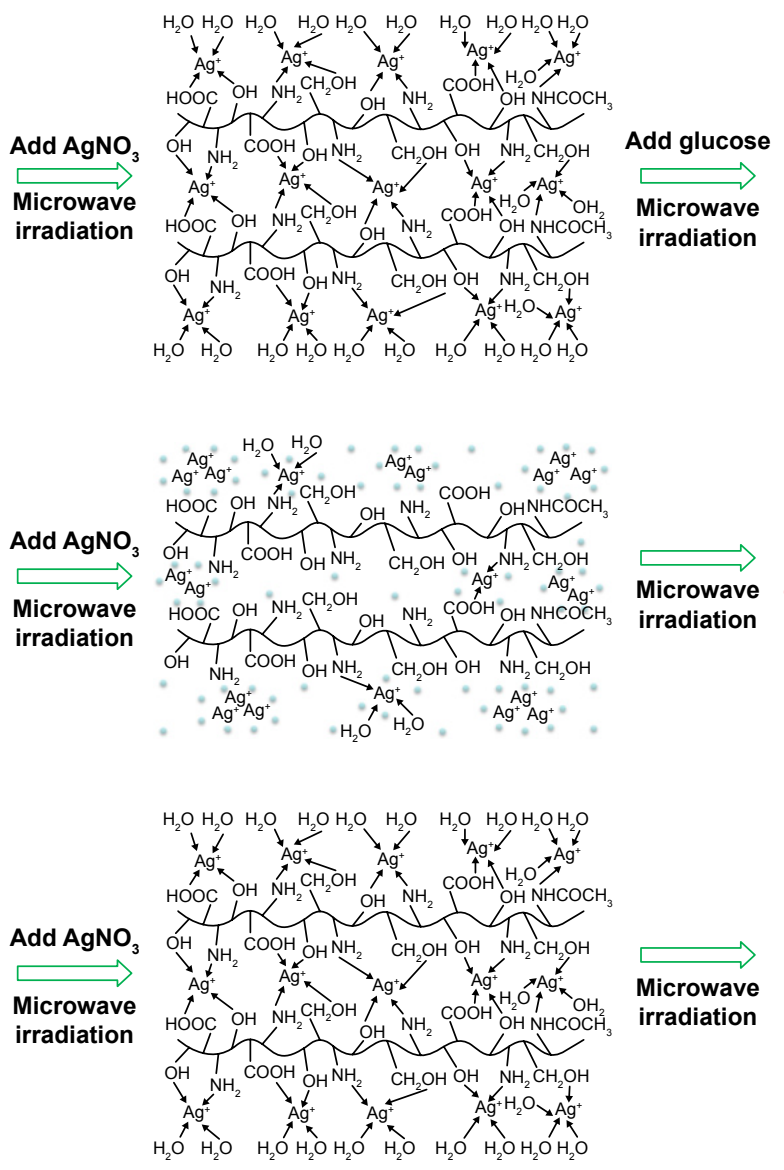

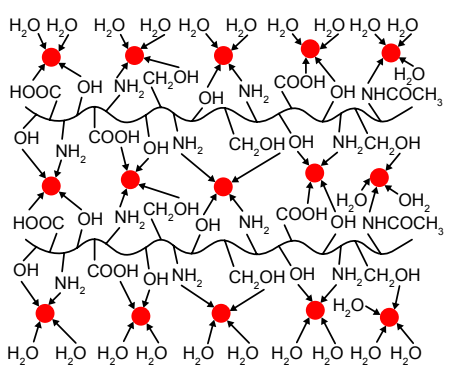

CMC-Ag1
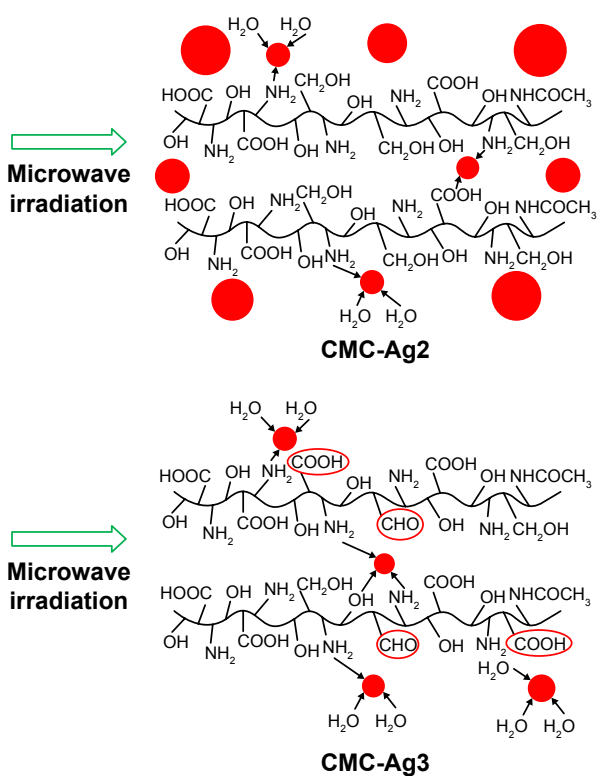

\section{$\longrightarrow$ CMC main chain $\mathrm{AgNPs} \cdot$ Glucose $\longrightarrow$ Coordination or electrostatic interaction}

Scheme I Schematic diagrams illustrating possible synthetic mechanisms of (A) CMC-AgI, (B) CMC-Ag2 and (C) CMC-Ag3.

Abbreviation: $\mathrm{CMC}-\mathrm{Ag}$, carboxymethyl chitosan-nanosilver.

crystallographic nature of the material. ${ }^{34}$ The XRD patterns of CMC-Ag1, CMC-Ag2, CMC-Ag3, and CMC are shown in Figure 2. Compared to pure $\mathrm{CMC}$, the obvious diffraction peaks at $2 \theta=38.03^{\circ}, 44.14^{\circ}, 64.47^{\circ}, 77.26^{\circ}$, and $81.35^{\circ}$ in the CMC-Ag hybrids (Figure 2 [a-c]) corresponded to the (111), (200), (220), (311), and (222) planes of silver crystals with a face centered cubic lattice structure (JCPDS 04-0783), which is consistent with our previous research..$^{29}$ These results indicate that $\mathrm{Ag}^{+}$was reduced to $\mathrm{Ag}^{0}$ and pure well-crystallized metallic silver was formed in all three synthetic protocols. According to the literature, ${ }^{35}$ the decreasing characteristic peak intensity of silver in the XRD patterns indicates that the amount of silver in hybrids decreased. Comparison of the diffraction intensity belonging to elemental silver in the XRD patterns of CMC-Ag1, CMC-Ag2, and CMC-Ag3 revealed that the silver content in CMC-Ag1 was the highest and in $\mathrm{CMC}-\mathrm{Ag} 3$ the lowest. Table 1 lists the amount of silver in $10 \mathrm{mg}$ of CMC-Ag1 $(510 \mu \mathrm{g}), \mathrm{CMC}-\mathrm{Ag} 2$ (407 $\mu \mathrm{g})$, and CMC-Ag3 $(98 \mu \mathrm{g})$ determined by quantitative

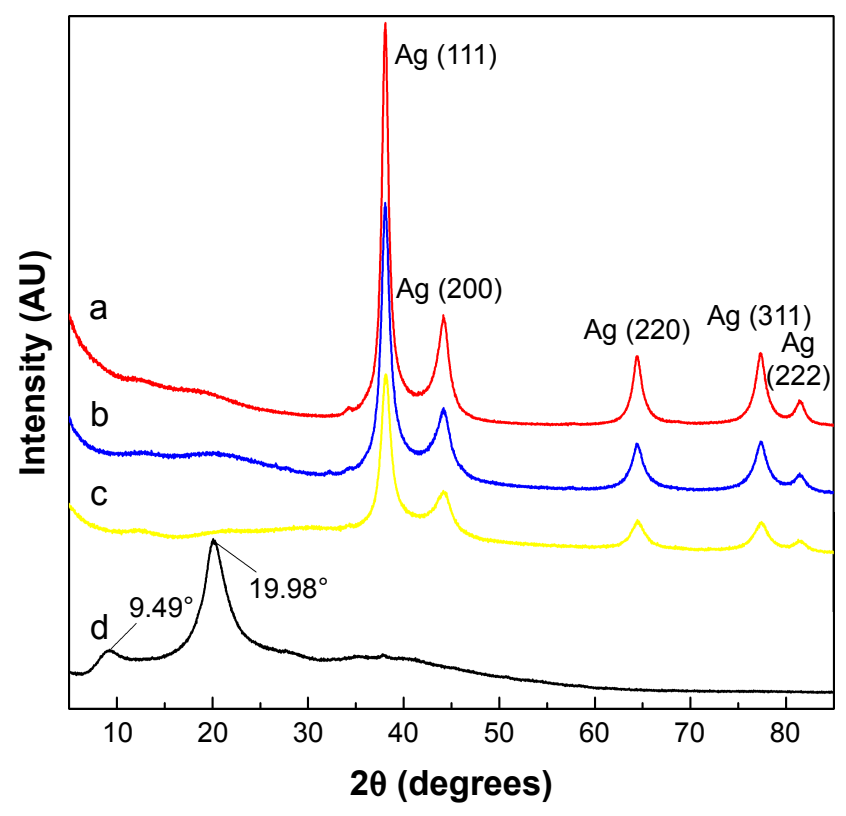

Figure 2 XRD patterns of CMC-AgI (a), CMC-Ag2 (b), CMC-Ag3 (c), and CMC (d) Abbreviations: XRD, X-ray diffraction; CMC-Ag, carboxymethyl chitosannanosilver. 
ICP-OES analyses, which further confirmed the findings of the XRD analysis.

UV-visible spectroscopy is widely used in the characterization of AgNPs, due to its excitation of surface plasmon resonance (SPR) in the UV-visible region (380-450 nm). ${ }^{37,41}$ Figure 3 shows the UV-visible spectra of the three CMC-Ag hybrids and pure $\mathrm{CMC}$. The $\mathrm{CMC}$ exhibited no peaks in the range of 300-600 nm in Figure 3 (d), but the three CMC-Ag hybrids (Figure 3 [a-c]) displayed unique SPR-absorption bands at around 403, 426, and $409 \mathrm{~nm}$, confirming the presence of AgNPs in the synthesized hybrids. A comparison of the absorption intensity of CMC-Ag1, CMC-Ag2, and CMC-Ag3 indicated that the addition of glucose resulted in a stronger SPR-absorption peak. This increase in SPRabsorption intensity can be attributed to greater AgNP synthesis. Zhao et al and other researchers have conducted theoretical studies on the correlation of UV-visible absorption with the size of metal spheres, and the general trend is that the SPR-absorption band shows a red shift with increasing particle size..$^{37,41,42}$ In the UV-visible spectra of CMC-Ag1, CMC-Ag2, and CMC-Ag3, a red shift of the SPR-absorption peak from 403 to $426 \mathrm{~nm}$ was related to the increasing particle size of AgNPs, suggesting that AgNPs were the smallest in CMC-Ag1. In addition, the corresponding SPR-absorption band of CMC-Ag1 was sharp and symmetrical, reflecting a very narrow distribution in the size of AgNPs. ${ }^{43}$ In contrast, the broad and asymmetric SPR-absorption band of CMC-Ag2 was indicative of wide particle-size distribution.

The morphology and dispersion of the AgNPs in hybrids were investigated using TEM. Figure $4 \mathrm{~A}$ displays the TEM image of CMC-Ag1: hundreds of spherical AgNPs of small size and uniform shape can be seen distributed

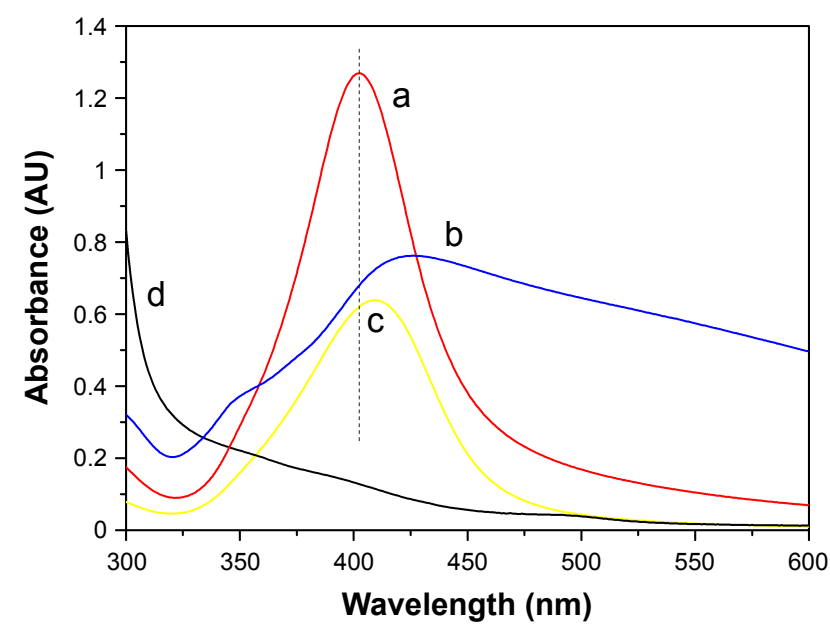

Figure 3 Ultraviolet-visible spectra of CMC-AgI (a), CMC-Ag2 (b), CMC-Ag3 (c), and $C M C$ (d).

Abbreviation: CMC-Ag, carboxymethyl chitosan-nanosilver. homogeneously in the CMC-polymer network. The inverse fast FT image (Figure 4G) and corresponding selected area electron-diffraction pattern (Figure $4 \mathrm{H}$ ) further indicated that AgNPs were crystalline in CMC-Ag1. The inverse fast FT image showed that the lattice-fringe spacing was $0.238 \mathrm{~nm}$, which was indexed to the $d$-spacing of the $\mathrm{Ag}$ (111) plane (JCPDS 04-0783). ${ }^{44}$ The selected area electrondiffraction pattern in Figure $4 \mathrm{H}$ showed periodic diffraction rings, indicating that the formed AgNPs were polycrystalline. Additionally, compared to Figure 4C, the amount of AgNPs in Figure 4A and B was obviously increased, indicating that adding glucose increased the amount of AgNPs, which was consistent with the XRD, ICP-OES and UV-visible results. By comparison with Figure 4A, large AgNPs with irregular shapes were unevenly dispersed along the surface of the CMC matrix, and appeared to agglomerate in certain regions in Figure 4B, demonstrating that the order of addition of glucose and $\mathrm{AgNO}_{3}$ had a significant effect on particle size and size distribution of the resulting AgNPs. The particle size and size distribution of the AgNPs in CMC-Ag1, CMC-Ag2, and CMC-Ag3 were measured as shown in Figure 4D-F. Comparing the three size-distribution histograms, obviously, the AgNPs in CMC-Ag1 had the smallest mean diameter $(12.22 \pm 2.57 \mathrm{~nm})$ and the narrowest size distribution (in the range of 6-20 nm).

In order to confirm further the formation mechanisms of the three CMC-Ag hybrids, the FT-IR spectra of CMC, CMC-Ag1, CMC-Ag2, and CMC-Ag3 were investigated as shown in Figure 5. In the characteristic peaks of CMC, the absorption peaks at around $3,431 \mathrm{~cm}^{-1}$ and $1,079 \mathrm{~cm}^{-1}$ corresponded to the coupled stretching vibrations of $\mathrm{N}-\mathrm{H}$ and $\mathrm{O}-\mathrm{H}$ groups and the $\mathrm{C}-\mathrm{O}$ stretching vibration of the secondary hydroxyl groups, which shifted to a lower frequency in the spectra of CMC-Ag1, CMC-Ag2, and CMC-Ag3, implying that $-\mathrm{NH}_{2},-\mathrm{NHCOCH}_{3}$, and $-\mathrm{OH}$ groups may be involved in silver attachment. Additionally, in Figure 5 (a), two peaks at around $1,610 \mathrm{~cm}^{-1}$ and $1,420 \mathrm{~cm}^{-1}$ were ascribed to the asymmetrical and symmetrical stretching vibrations of the carboxyl groups, while the red shift of these peaks in Figure $5(\mathrm{~b}-\mathrm{d})$ suggested that the $-\mathrm{COOH}$ groups may also contribute to silver immobilization. By comparing the spectra of the three hybrids with CMC, the extent of red shift of these characteristic peaks in CMC-Ag1 was the largest, while the least was in $\mathrm{CMC}-\mathrm{Ag} 2$, indicating strong coordination and electrostatic interaction between CMC and silver in CMC-Ag1, but extremely weak interaction in CMC-Ag2. Additionally, in the spectrum of CMC-Ag3, the stronger peaks at $1,593 \mathrm{~cm}^{-1}$ and $1,405 \mathrm{~cm}^{-1}$ corresponded to 
A
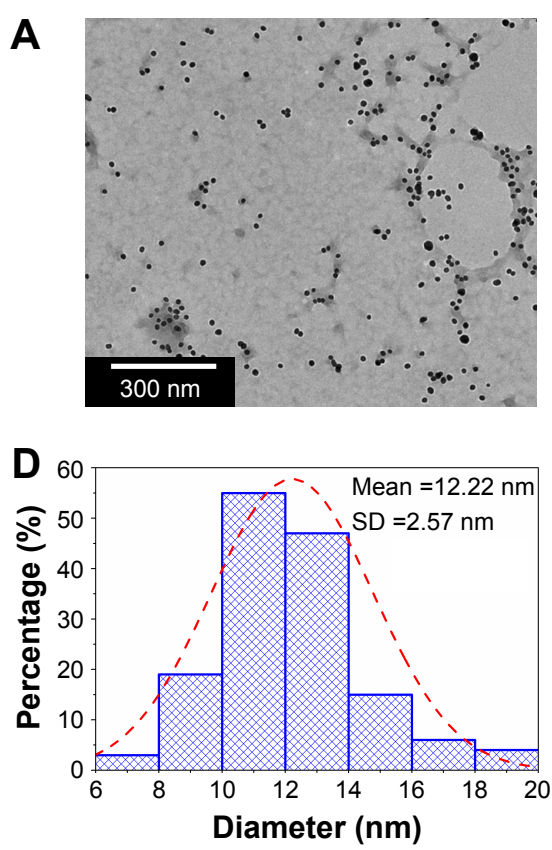

B
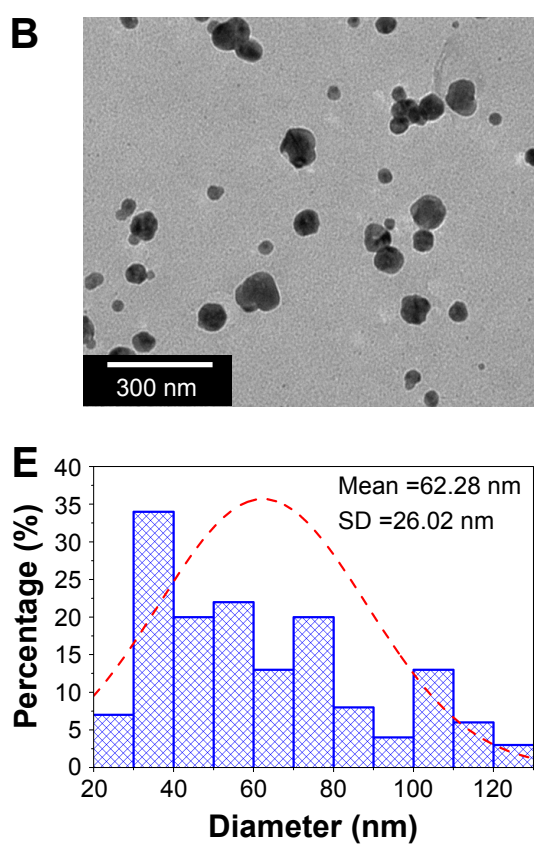

C
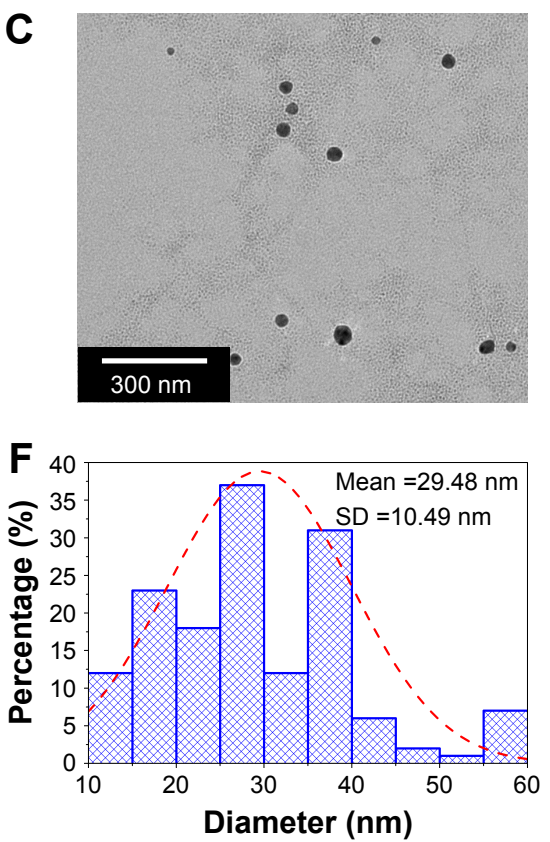

G

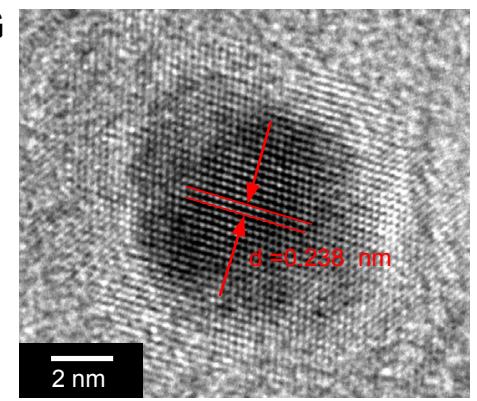

$\mathbf{H}$

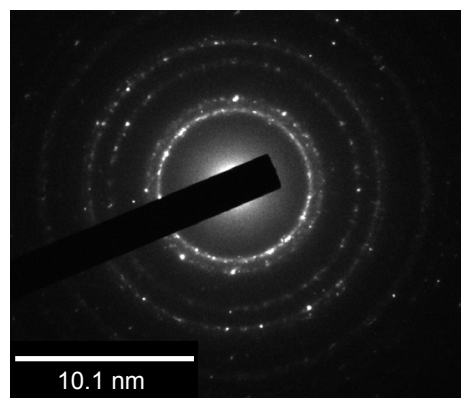

Figure 4 Morphology and dispersion of AgNPs in CMC-AgI, CMC-Ag2 and CMC-Ag3.

Notes: (A-C) TEM images of CMC-AgI, CMC-Ag2, and CMC-Ag3; (D-F) size-distribution histograms of AgNPs in CMC-AgI, CMC-Ag2, and CMC-Ag3, respectively; $(\mathbf{G}, \mathbf{H})$ IFFT image and SAED pattern of CMC-AgI.

Abbreviations: TEM, transmission electron microscopy; CMC-Ag, carboxymethyl chitosan-nanosilver; SD, standard deviation; IFFT, inverse fast Fourier transform; SAED, selected area electron diffraction.

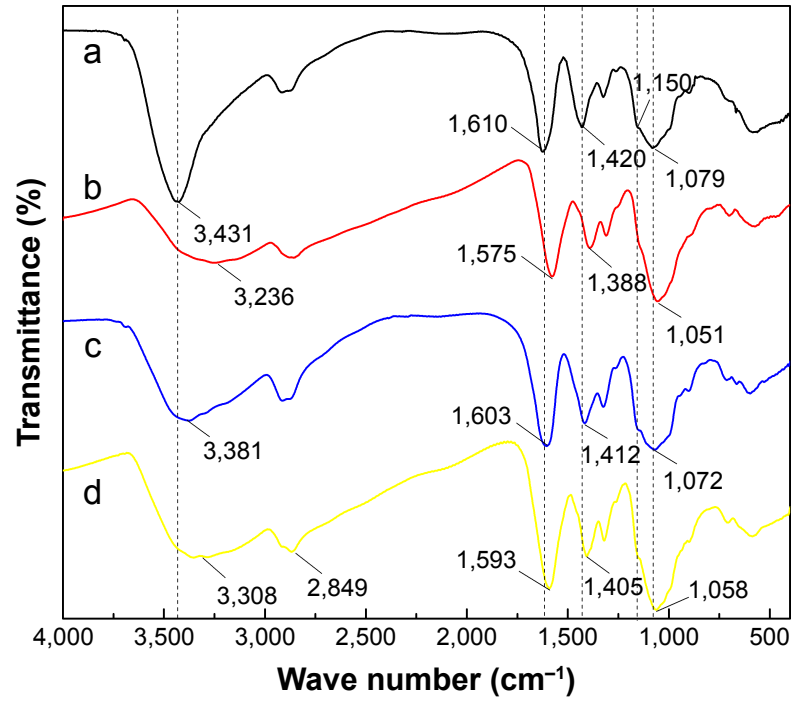

Figure 5 FT-IR spectra of CMC (a), CMC-AgI (b), CMC-Ag2 (c), and CMC-Ag3 (d). Abbreviations: FT-IR, Fourier-transform infrared; CMC, carboxymethyl chitosan; CMC-Ag, CMC-nanosilver. the $-\mathrm{COOH}$ groups and a new adsorption band at $2,849 \mathrm{~cm}^{-1}$ assigned to the $\mathrm{C}-\mathrm{H}$ stretching vibration of the $-\mathrm{CHO}$ groups appeared, confirming that $\mathrm{CMC}$ can be used as a reducing agent by oxidizing the primary alcohol to aldehyde or carboxyl groups without an additional reducing reagent. According to these results, the synthetic mechanisms of the three hybrids were verified, and are consistent with Scheme 1.

\section{Analysis of antimicrobial activity}

Table 2 lists the MIC results of CMC, CMC-Ag1, CMC-Ag2, CMC-Ag3, and commercially available AgNPs against the Gram-positive $S$. aureus and Gram-negative E. coli. As shown in Table 2, pure CMC showed weak inhibition of $S$. aureus and E. coli, but commercially available AgNPs displayed relatively strong antibacterial properties. Since CMC-Ag hybrids can combine the intrinsic advantages of the CMC matrix and AgNPs to show synergistic antibacterial effects, ${ }^{44}$ 
Table 2 MIC results of CMC, CMC-AgI, CMC-Ag2, CMC-Ag3, and commercially available AgNPs

\begin{tabular}{lll}
\hline Sample code & MIC values $(\%, w / v)$ & \\
\cline { 2 - 2 } & Staphylococcus aureus & Escherichia coli \\
\hline Blank & $-^{\mathrm{a}}$ & $-^{\mathrm{a}}$ \\
PBS & $-^{\mathrm{a}}$ & $-^{\mathrm{a}}$ \\
CMC & 2 & 3 \\
CMC-AgI & 0.00625 & 0.0125 \\
CMC-Ag2 & 0.025 & 0.05 \\
CMC-Ag3 & 0.0125 & 0.025 \\
AgNPs & 0.025 & 0.075 \\
\hline
\end{tabular}

Notes: ${ }^{a} \mathrm{No}$ antibacterial properties. All experiments conducted in triplicate. Abbreviations: MIC, minimum inhibitory concentration; CMC, carboxymethyl chitosan; CMC-Ag, CMC-nanosilver; AgNPs, silver nanoparticles; PBS, phosphatebuffered saline.

it is not surprising that their antimicrobial activities were superior to $\mathrm{CMC}$ alone and AgNPs. For the three hybrids, the $\mathrm{MIC}$ values of CMC-Ag1 were much lower, indicating that $\mathrm{CMC}-\mathrm{Ag} 1$ exhibited more outstanding inhibition of bacteria than CMC-Ag2 and CMC-Ag3.

Furthermore, it can be seen from Table 2 that overall, samples exhibited better antimicrobial activities against $S$. aureus than E. coli. Figure 6 displays the morphology of $S$. aureus and $E$. coli colonies after treatment with CMC-Ag1, CMC-Ag2, and CMC-Ag3 at a concentration of $0.00625 \%$ compared to the blank control. As shown in Figure 6B and F, CMC-Ag1 completely inhibited S. aureus growth, but showed very little effect on the growth of $E$. coli at the same concentration. According to the literature, ${ }^{45-47}$ this result may be attributed to the different cell-wall structure of the two types of bacteria. S. aureus, a typical Gram-positive bacterium, has a thick cell wall that is composed mainly of peptidoglycan. The peptidoglycan layer has a netted structure with many pores that do not effectively block the invasion of antibacterial agents. However, the cell wall of E. coli, a typical Gram-negative bacterium, is composed of a thin peptidoglycan layer and an outer membrane. The outer membrane, consisting in lipopolysaccharide, lipoprotein, and phospholipids acts as a solid barrier, resisting the intrusion of "foreign" molecules. Therefore, antibacterial agents are more effective in inhibiting the growth of $S$. aureus than E. coli.

\section{In vitro silver-release study}

The in vitro release profiles of silver from the CMC-Ag hybrids prepared using the three synthetic protocols are presented in Figure 7. As shown in Figure 7A, CMC-Ag1 exhibited stable and continuous silver release with no obvious initial burst. This is because in CMC-Ag1, well-dispersed AgNPs were firmly anchored and embedded inside the CMC-polymer network, and the strong interactions between silver and CMC prevented the dissolution of AgNPs to silver ions and the diffusion of silver ions into phosphate buffer, allowing CMC-Ag1 to release silver in a controlled way. ${ }^{48}$ In contrast, the silver-release profiles of CMC-Ag2 and $\mathrm{CMC}-\mathrm{Ag} 3$ can be described as a two-step process of an initial burst release followed by subsequent slower release. Specifically, CMC-Ag2 showed very fast silver release in the
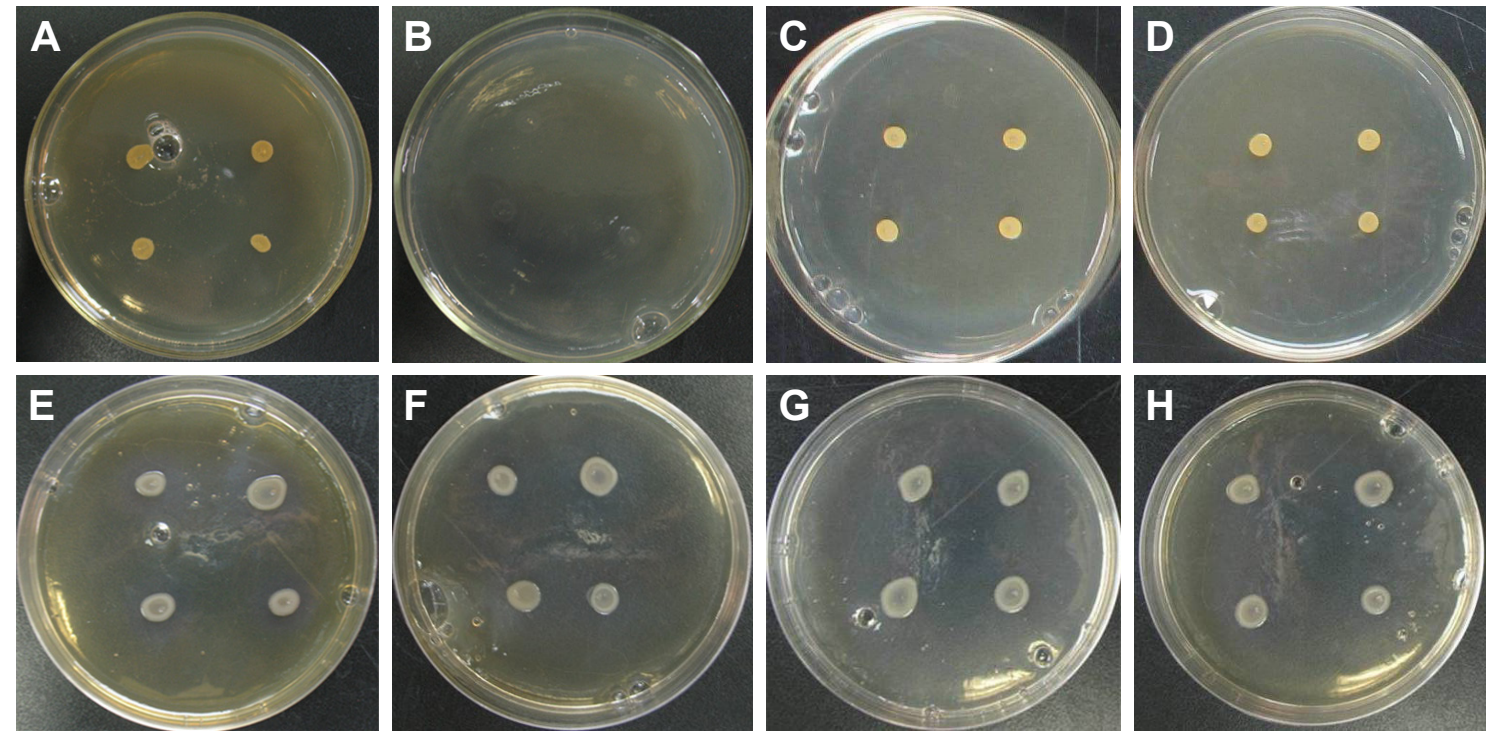

Figure 6 Colonies of Staphylococcus aureus and Escherichia coli after treatment with CMC-AgI, CMC-Ag2, and CMC-Ag3 at a concentration of 0.00625\%.

Notes: (A-D) Colonies of S. aureus after treatment with blank, CMC-AgI, CMC-Ag2, and CMC-Ag3, respectively; (E-H) colonies of E. coli after treatment with blank, CMC-AgI, CMC-Ag2, and CMC-Ag3, respectively.

Abbreviation: CMC-Ag, carboxymethyl chitosan-nanosilver. 

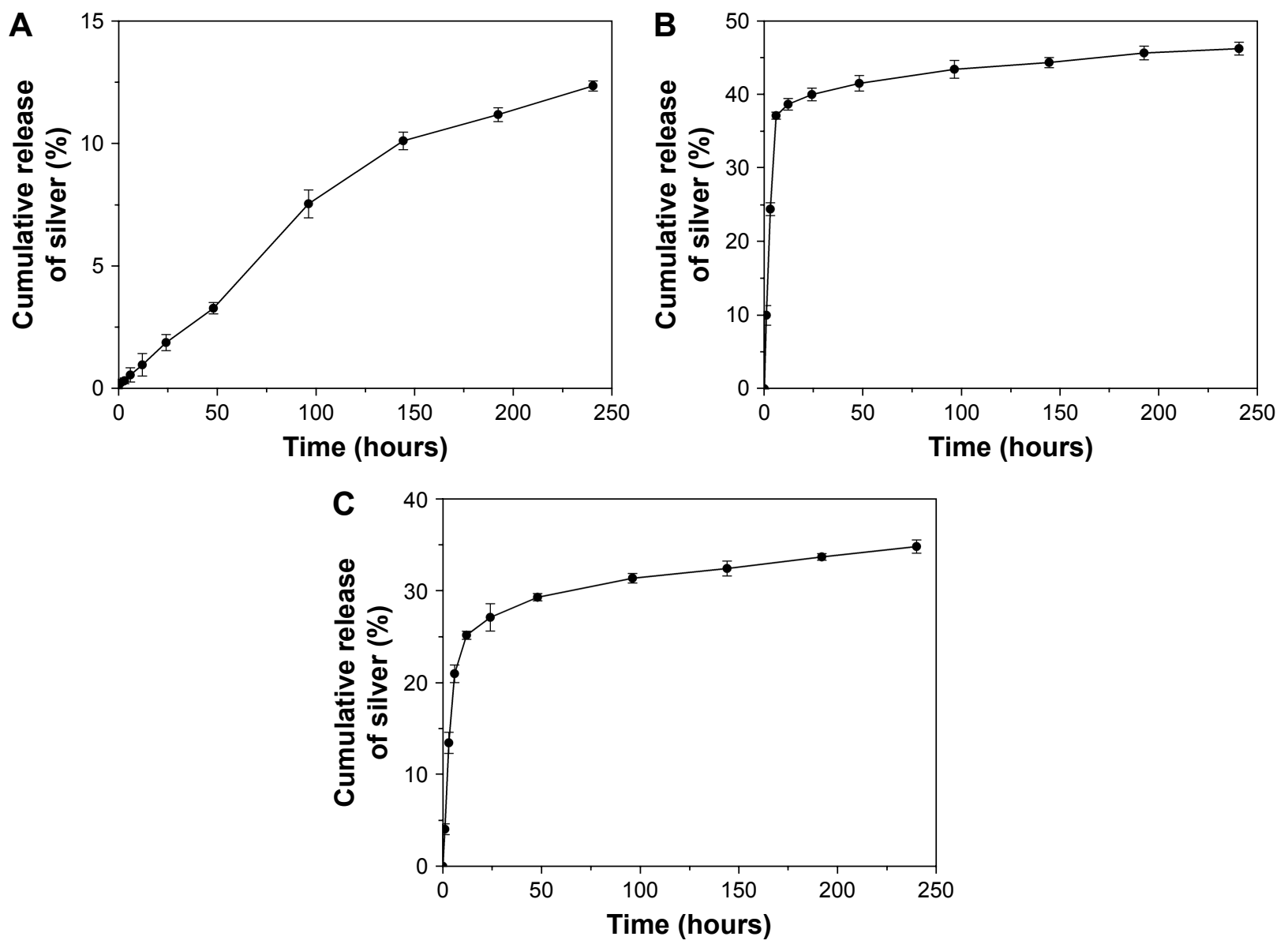

Figure 7 In vitro silver release time profiles of CMC-AgI (A), CMC-Ag2 (B), and CMC-Ag3 (C).

Notes: All experiments were conducted in triplicate. Error bars represent standard deviation.

Abbreviation: CMC-Ag, carboxymethyl chitosan-nanosilver.

early stage (up to 6 hours), then the release rate slowed in the second stage. For CMC-Ag3, the release amount of silver increased over the first 12 hours, and then slowed at longer times. The initial burst release could be attributed to the rapid dissolution of free AgNPs suspended in the phosphate buffer, as well as the discrete AgNPs attached on the surface of CMC. ${ }^{4}$ In addition, as shown in Table 1, the cumulative percentage of silver released after 240 hours was much lower for CMC-Ag1 (12.35\%) than for CMC-Ag2 (46.19\%) and CMC-Ag3 (34.69\%), suggesting that CMC-Ag1 had much better performance in retaining silver for prolonged periods. Besides, in the case of CMC-Ag1, the total concentration of silver released after 240 hours was $6.3 \mathrm{ppm}$, which was lower than the half maximal inhibitory concentration (15-21 ppm) of silver ions reported for some mammalian cells, such as L929 murine fibroblasts, ${ }^{17,49}$ implying that CMC-Ag1 exhibited good biocompatibility with mammalian cells. This may help expand its potential applications in the biomedical and biological fields.

\section{Conclusion}

A green, simple and efficient approach for the fabrication of CMC-Ag hybrids was reported. The synthesis was conducted in an aqueous medium in the presence of microwave irradiation for 10 minutes using different synthetic protocols. Spherical AgNPs were successfully obtained via all three protocols, but the amount, average size, and size distribution of AgNPs in the different hybrids varied considerably. Results showed that the order of addition of glucose and $\mathrm{AgNO}_{3}$ had a significant effect on the particle size and size distribution of AgNPs, and the addition of glucose was conducive to the production of more AgNPs. With the use of glucose as the reducing agent (adding glucose after $\mathrm{AgNO}_{3}$ ), many stable, uniform, and small AgNPs were synthesized in situ on the CMC chains. The size distribution of AgNPs was quite narrow, in the range of $6-20 \mathrm{~nm}$, and the mean diameter was only $12.22 \pm 2.57 \mathrm{~nm}$.

Compared to CMC alone and commercially available AgNPs, the antimicrobial activities of the three hybrids were 
significantly better. Of the three hybrids, CMC-Ag1 exhibited superior antimicrobial activity to $\mathrm{CMC}-\mathrm{Ag} 2$ and CMC-Ag3. Additionally, for CMC-Ag1, well-dispersed AgNPs were firmly anchored and embedded inside the CMC-polymer network. The strong coordination and electrostatic interactions between $\mathrm{CMC}$ and silver and the good steric protection provided by CMC enabled CMC-Ag1 to display stable and continuous silver release and show better silver retention compared to CMC-Ag2 and CMC-Ag3.

Overall, CMC-Ag1 exhibited outstanding hybrid performance with excellent antimicrobial activity, as well as sustained and controlled silver release, suggesting that this novel material may be a promising antimicrobial candidate for application in biomedical and biological fields, coatings, clothing, textiles, wood products, food packaging, and other applications. Further investigation into CMC-Ag1, including the kinetics of silver release and the mechanism of antimicrobial activity, will be initiated in the near future.

\section{Acknowledgments}

The authors would like to acknowledge the Fundamental Research Fund for Central Universities (2016ZCQ01 and 2016BLRD03) for financial support. The authors express their thanks to Dr Lanxing Du for her contributions in proofreading the manuscript.

\section{Disclosure}

The authors report no conflicts of interest in this work.

\section{References}

1. He W, Kim HK, Wamer WG, Melka D, Callahan JH, Yin JJ. Photogenerated charge carriers and reactive oxygen species in $\mathrm{ZnO} / \mathrm{Au}$ hybrid nanostructures with enhanced photocatalytic and antibacterial activity. J Am Chem Soc. 2013;136(2):750-757.

2. Hasan J, Crawford RJ, Ivanova EP. Antibacterial surfaces: the quest for a new generation of biomaterials. Trends Biotechnol. 2013;31(5): 295-304.

3. Tan P, Li YH, Liu XQ, Jiang Y, Sun LB. Core-shell AgCl@SiO nanoparticles: $\mathrm{Ag}(\mathrm{I})$-based antibacterial materials with enhanced stability. ACS Sustain Chem Eng. 2016;4(6):3268-3275.

4. Silva FM, Pinto RJ, Daniel-da-Silva AL, Trindade T. Cationic release behaviour of antimicrobial cellulose/silver nanocomposites. Cellulose. 2014;21(5):3551-3560.

5. Li P, Poon YF, Li WF, et al. A polycationic antimicrobial and biocompatible hydrogel with microbe membrane suctioning ability. Nat Mater. 2011;10(2):149-156.

6. Muñoz-Bonilla A, Fernández-García M. The roadmap of antimicrobial polymeric materials in macromolecular nanotechnology. Eur Polym J. 2015;65:46-62.

7. Rai M, Yadav A, Gade A. Silver nanoparticles as a new generation of antimicrobials. Biotechnol Adv. 2009;27(1):76-83.

8. Eckhardt S, Brunetto PS, Gagnon J, Priebe M, Giese B, Fromm KM. Nanobio silver: its interactions with peptides and bacteria, and its uses in medicine. Chem Rev. 2013;113(7):4708-4754.
9. Wan G, Ruan L, Yin Y, et al. Effects of silver nanoparticles in combination with antibiotics on the resistant bacteria Acinetobacter baumannii. Int J Nanomedicine. 2016;11:3789-3800.

10. Chopra I. The increasing use of silver-based products as antimicrobial agents: a useful development or a cause for concern? J Antimicrob Chemother. 2007;59(4):587-590.

11. Ramalingam B, Khan MM, Mondal B, Mandal AB, Das SK. Facile synthesis of silver nanoparticles decorated magnetic-chitosan microsphere for efficient removal of dyes and microbial contaminants. ACS Sustain Chem Eng. 2015;3(9):2291-2302.

12. Banasiuk R, Frackowiak JE, Krychowiak M, et al. Synthesis of antimicrobial silver nanoparticles through a photomediated reaction in an aqueous environment. Int J Nanomedicine. 2016;11:315-324.

13. Arokiyaraj S, Arasu MV, Vincent S, et al. Rapid green synthesis of silver nanoparticles from Chrysanthemum indicum $\mathrm{L}$ and its antibacterial and cytotoxic effects: an in vitro study. Int J Nanomedicine. 2014;9: 379-388.

14. Liu J, Sonshine DA, Shervani S, Hurt RH. Controlled release of biologically active silver from nanosilver surfaces. ACS Nano. 2010;4(11): 6903-6913.

15. Benn TM, Westerhoff P. Nanoparticle silver released into water from commercially available sock fabrics. Environ Sci Technol. 2008;42(11): 4133-4139.

16. Hussain SM, Schlager JJ. Safety evaluation of silver nanoparticles: inhalation model for chronic exposure. Toxicol Sci. 2009;108(2): 223-224.

17. Gagnon J, Clift MJ, Vanhecke D, et al. Synthesis, characterization, antibacterial activity and cytotoxicity of hollow $\mathrm{TiO}_{2}$-coated $\mathrm{CeO}_{2}$ nanocontainers encapsulating silver nanoparticles for controlled silver release. J Mater Chem B Mater Biol Med. 2016;4(6):1166-1174.

18. Park SY, Chung JW, Priestley RD, Kwak SY. Covalent assembly of metal nanoparticles on cellulose fabric and its antimicrobial activity. Cellulose. 2012;19(6):2141-2151.

19. Raveendran P, Fu J, Wallen SL. A simple and "green" method for the synthesis of $\mathrm{Au}, \mathrm{Ag}$, and $\mathrm{Au}-\mathrm{Ag}$ alloy nanoparticles. Green Chem. 2006;8(1):34-38.

20. Hebbalalu D, Lalley J, Nadagouda MN, Varma RS. Greener techniques for the synthesis of silver nanoparticles using plant extracts, enzymes, bacteria, biodegradable polymers, and microwaves. ACS Sustain Chem Eng. 2013;1(7):703-712.

21. Kahrilas GA, Haggren W, Read RL, et al. Investigation of antibacterial activity by silver nanoparticles prepared by microwave-assisted green syntheses with soluble starch, dextrose, and arabinose. ACS Sustain Chem Eng. 2014;2(4):590-598.

22. Bazant P, Kuritka I, Munster L, Kalina L. Microwave solvothermal decoration of the cellulose surface by nanostructured hybrid $\mathrm{Ag} / \mathrm{ZnO}$ particles: a joint XPS, XRD and SEM study. Cellulose. 2015;22(2): $1275-1293$

23. Wang Y, Lin F, Zhao Y, et al. The sustained-release behavior and in vitro and in vivo transfection of pEGFP-loaded core-shell-structured chitosanbased composite particles. Int J Nanomedicine. 2014;9:4965-4978.

24. Wang LS, Wang CY, Yang CH, et al. Synthesis and anti-fungal effect of silver nanoparticles-chitosan composite particles. Int J Nanomedicine. 2015;10:2685-2696.

25. Long Y, Ran X, Zhang L, et al. A method for the preparation of silver nanoparticles using commercially available carboxymethyl chitosan and sunlight. Mater Lett. 2013;112:101-104.

26. An NT, Dong NT, Hanh PT, et al. Silver-N-carboxymethyl chitosan nanocomposites: synthesis and its antibacterial activities. J Bioterror Biodef. 2010;1(1):102.

27. Laudenslager MJ, Schiffman JD, Schauer CL. Carboxymethyl chitosan as a matrix material for platinum, gold, and silver nanoparticles. Biomacromolecules. 2008;9(10):2682-2685.

28. Huang S, Yu Z, Qi C, Zhang Y. Chitosan/organic rectorite nanocomposites rapidly synthesized by microwave irradiation: effects of chitosan molecular weight. $R S C A d v .2015 ; 5(104): 85272-85279$. 
29. Huang S, Wang J, Zhang Y, Yu Z, Qi C. Quaternized carboxymethyl chitosan-based silver nanoparticles hybrid: microwave-assisted synthesis, characterization and antibacterial activity. Nanomaterials. 2016; 6(6): 118 .

30. Hosseini SF, Zandi M, Rezaei M, Farahmandghavi F. Two-step method for encapsulation of oregano essential oil in chitosan nanoparticles: preparation, characterization and in vitro release study. Carbohydr Polym. 2013;95(1):50-56.

31. Csóka L, Božanić DK, Nagy V, et al. Viscoelastic properties and antimicrobial activity of cellulose fiber sheets impregnated with Ag nanoparticles. Carbohydr Polym. 2012;90(2):1139-1146.

32. Barud HS, Barrios C, Regiani T, et al. Self-supported silver nanoparticles containing bacterial cellulose membranes. Mat Sci Eng C Mater Biol Appl. 2008;28(4):515-518.

33. Cai J, Kimura S, Wada M, Kuga S. Nanoporous cellulose as metal nanoparticles support. Biomacromolecules. 2008;10(1):87-94.

34. Dong YY, Fu LH, Liu S, Ma MG, Wang B. Silver-reinforced cellulose hybrids with enhanced antibacterial activity: synthesis, characterization, and mechanism. RSC Adv. 2015;5(118):97359-97366.

35. Li Z, Wang L, Chen S, et al. Facilely green synthesis of silver nanoparticles into bacterial cellulose. Cellulose. 2014;22(1):373-383.

36. Zhang M, Zhang Y, Helleur R. Selective adsorption of $\mathrm{Ag}^{+}$by ionimprinted O-carboxymethyl chitosan beads grafted with thioureaglutaraldehyde. Chem Eng J. 2015;264:56-65.

37. Zhao X, Xia Y, Li Q, et al. Microwave-assisted synthesis of silver nanoparticles using sodium alginate and their antibacterial activity. Colloids Surf A Physicochem Eng Asp. 2014;444:180-188.

38. Fu LH, Deng F, Ma MG, Yang J. Green synthesis of silver nanoparticles with enhanced antibacterial activity using holocellulose as a substrate and reducing agent. RSC Adv. 2016;6(34):28140-28148.

39. Emam HE, Ahmed HB. Polysaccharides templates for assembly of nanosilver. Carbohydr Polym. 2016;135:300-307.
40. El-Naggar ME, Shaheen TI, Fouda MM, Hebeish AA. Eco-friendly microwave-assisted green and rapid synthesis of well-stabilized gold and core-shell silver-gold nanoparticles. Carbohydr Polym. 2016;136: 1128-1136.

41. Aladpoosh R, Montazer M, Samadi N. In situ green synthesis of silver nanoparticles on cotton fabric using Seidlitzia rosmarinus ashes. Cellulose. 2014;21(5):3755-3766.

42. Lee HJ, Lee SG, Oh EJ, et al. Antimicrobial polyethyleneiminesilver nanoparticles in a stable colloidal dispersion. Colloids Surf B Biointerfaces. 2011;88(1):505-511.

43. An J, Ji Z, Wang D, Luo Q, Li X. Preparation and characterization of uniform-sized chitosan/silver microspheres with antibacterial activities. Mater Sci Eng C Mater Biol Appl. 2014;36:33-41.

44. Mallick S, Sanpui P, Ghosh SS, Chattopadhyay A, Paul A. Synthesis, characterization and enhanced bactericidal action of a chitosan supported core-shell copper-silver nanoparticle composite. RSC Adv. 2015; 5(16):12268-12276.

45. Hassabo AG, Nada AA, Ibrahim HM, Abou-Zeid NY. Impregnation of silver nanoparticles into polysaccharide substrates and their properties. Carbohydr Polym. 2015;122:343-350.

46. Kong M, Chen XG, Liu CS, Liu CG, Meng XH, Yu LJ. Antibacterial mechanism of chitosan microspheres in a solid dispersing system against E. coli. Colloids Surf B Biointerfaces. 2008;65(2):197-202.

47. Sun L, Du Y, Fan L, Chen X, Yang J. Preparation, characterization and antimicrobial activity of quaternized carboxymethyl chitosan and application as pulp-cap. Polymer. 2006;47(6):1796-1804.

48. Sámano-Valencia C, Martínez-Castañón G, Martínez-Martínez R, et al. Bactericide efficiency of a combination of chitosan gel with silver nanoparticles. Mater Lett. 2013;106:413-416.

49. Müller G, Kramer A. Biocompatibility index of antiseptic agents by parallel assessment of antimicrobial activity and cellular cytotoxicity $J$ Antimicrob Chemother. 2008;61(6):1281-1287.
International Journal of Nanomedicine

\section{Publish your work in this journal}

The International Journal of Nanomedicine is an international, peerreviewed journal focusing on the application of nanotechnology in diagnostics, therapeutics, and drug delivery systems throughout the biomedical field. This journal is indexed on PubMed Central, MedLine, CAS, SciSearch $\AA$, Current Contents $₫ /$ Clinical Medicine,

\section{Dovepress}

Journal Citation Reports/Science Edition, EMBase, Scopus and the Elsevier Bibliographic databases. The manuscript management system is completely online and includes a very quick and fair peer-review system, which is all easy to use. Visit http://www.dovepress.com/ testimonials.php to read real quotes from published authors. 